\title{
Genetic loci for resistance to podocyte injury caused by the tensin 2 gene deficiency in mice
}

\author{
Yuki Takahashi, Hayato Sasaki ${ }^{*}$, Shiori Okawara and Nobuya Sasaki
}

\begin{abstract}
Background: Tensin2 is a focal adhesion-localized multidomain protein expressed in various tissues, and its dysfunction leads to alterations in podocytes. However, these podocyte-related manifestations are dependent on murine strain. Tensin2 dysfunction results in susceptible strains developing podocyte foot process effacement and massive albuminuria, whereas podocytes in resistant strains remain almost intact. In our previous studies, quantitative trait loci analysis and congenic analysis using resistant C57BL/6J and susceptible ICGN mice identified a modifier locus associated with podocyte injury caused by tensin2 dysfunction on chromosome 2 . However, the effect of this modifier locus on chromosome 2 is insufficient to explain the resistance of $C 57 \mathrm{BL} / 6 \mathrm{~J}$ mice to tensin2 dysfunction, indicating the existence of other modifier genes.

Results: Whereas previous studies focused on the severity of chronic kidney disease, the present study focused on podocyte injury. We performed a genome-wide linkage analysis of backcrosses between two tensin2-deficient mouse strains, B6.ICGN-Tns $2^{\text {nph }}$ and FVB.ICGN-Tns $2^{\text {nph }}$, and detected a novel major modifier locus on chromosome 10. The combined effect of the C57BL/6J alleles of the two loci on chromosomes 2 and 10 reduced the urinary albumin excretion caused by tensin2 dysfunction to a level comparable to that of C57BL/6J mice.

Conclusions: These data indicate that the resistance to podocyte injury caused by tensin2 dysfunction is mainly produced by the effects of the modifier genes on the two loci. The identification of these modifier genes is expected to help elucidate the mechanism underlying podocyte injury.
\end{abstract}

Keywords: Tensin2, Albuminuria, Podocyte, Linkage analysis

\section{Background}

Tensin2 (Tns2), a member of the tensin family that includes Tns1, Tns3 and Tns4, is a focal adhesionlocalized multidomain protein that possesses protein tyrosine phosphatase (PTP) and Src homology 2 (SH2)-phosphotyrosine binding (PTB) domains [1]. Tns2 mRNA is detected in the brain, heart, kidney, skeletal muscle, liver, lung, colon, small intestine and other tissues [2-6]. Stable expression of Tns2 in HEK293 cells alters cell morphology and reduces actin filaments. Overexpression of Tns2 in HEK293 cells reduces cell proliferation in a functional PTP domain-dependent manner and inhibits cell migration [7]. In contrast,

\footnotetext{
* Correspondence: hsasaki@vmas.kitasato-u.ac.jp

Laboratory of Laboratory Animal Science and Medicine, School of Veterinary

Medicine, Kitasato University, 35-1, Higashi-23, Towada, Aomori 034-8628, Japan
}

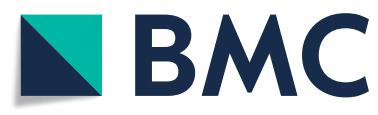

(C) The Author(s). 2018 Open Access This article is distributed under the terms of the Creative Commons Attribution 4.0 International License (http://creativecommons.org/licenses/by/4.0/), which permits unrestricted use, distribution, and reproduction in any medium, provided you give appropriate credit to the original author(s) and the source, provide a link to the Creative Commons license, and indicate if changes were made. The Creative Commons Public Domain Dedication waiver (http://creativecommons.org/publicdomain/zero/1.0/) applies to the data made available in this article, unless otherwise stated.

stable expression of Tns2 protein with its $C$ terminus fused to green fluorescent protein in HEK293 cells promotes cell migration [2]. In a podocyte cell line, it was shown that Tns 2 knockdown enhances actin stress fiber formation and cell migration [8]. Taken together, these studies suggest that Tns2 function is involved in the reorganization of the actin cytoskeleton.

However, in vivo, Tns2 dysfunction only leads to apparent abnormalities in the kidney glomerulus [4, 8]. Newborn ICGN mice, carrying a Tns2-null mutation (hereafter $n p h$ ), showed normal glomerular development, whereas podocyte foot process effacement and abnormal thickening of the glomerular basement membrane (GBM) were observed by 14 days after birth [9]. GBM thickening, which begins during glomerular maturation in ICGN mice, is considered to be a result of the abnormal extracellular matrix accumulation caused 
by podocytes but not by endothelial cells [9]. As observed in chronic kidney disease, ICGN mice then develop glomerular injury, including mesangial expansion, and massive albuminuria followed by tubulointerstitial injury with age $[10,11]$.

The podocyte-related manifestations of Tns2 dysfunction also depend on murine strain. For example, in response to $n p h$ mutation, albuminuria or podocyte foot process effacement was observed in $\mathrm{FVB} / \mathrm{NJ}(\mathrm{FVB})$ and $\mathrm{DBA} / 2 \mathrm{~J}$, but not in $\mathrm{C} 57 \mathrm{BL} / 6 \mathrm{~J}$ (B6) and $129^{+ \text {Ter }} / \mathrm{SvJcl}$ mice [12-16]. This disparity among strains indicates the presence of modifier genes. A comparison of the differences in the genetic backgrounds between the Tns2-deficiency resistant and susceptible strains can help in identifying these modifier genes, which may also help in elucidating the mechanism underlying Tns2 function in podocytes. Indeed, we previously performed quantitative trait loci (QTL) analysis and congenic analysis using resistant B6 and susceptible ICGN mice, and identified a modifier locus associated with podocyte injury caused by the nph mutation, Tpir, on chromosome $2[17,18]$. ICGN.B6 congenic strains carrying the B6 allele of Tpir showed alleviated podocyte foot process effacement, GBM thickening and albuminuria, although the degree of improvement was inferior to that in the B6 genetic background itself, which shows almost no podocyte alteration [18]. This result indicates the existence of other modifier genes. The following points may explain why our previous QTL analysis failed to find the other significant loci: (1) our previous analysis focused on the severity of chronic kidney disease, and evaluated the traits related to the late-phase of chronic kidney disease in 16-week-old mice, such as tubulointerstitial injury, renal anemia and renal failure, but not albuminuria; (2) the quantitative traits, except for the histological scores, varied greatly in the ICGN mice themselves; and (3) the glomerular injury score focused on mesangial matrix accumulation, which is primarily caused by abnormal extracellular matrix metabolism in mesangial cells [19]. A further problem is that complete genetic information on the ICGN mouse genome remains unavailable.

In this study, we focused on podocyte injury and undertook a genome-wide linkage analysis of backcrosses between two Tns2-deficient mouse strains, B6.ICGNTns2 $2^{n p h} \quad\left(\mathrm{~B} 6-T n s 2^{n p h}\right)$ and FVB.ICGN-Tns2 $2^{n p h}$ (FVB$T n s 2^{n p h}$ ) to identify modifier loci that may have been missed in previous studies.

\section{Methods \\ Animals}

B6-Tns $2^{\text {nph }}$ and FVB-Tns2 $2^{\text {nph }}$ congenic mice were generated as described previously $[12,15] . \mathrm{B} 6 \times \mathrm{FVB} \mathrm{F}_{1}$ hybrid Tns $2^{n p h}$ (F1) mice were obtained by mating female B6-Tns $2^{n p h}$ mice to male FVB-Tns2 ${ }^{n p h}$ mice. N2 backcross Tns $2^{\text {nph }}$ progenies were then obtained by mating female F1 mice to male FVB-Tns $2^{n p h}$ mice (Fig. 1a). The SPF
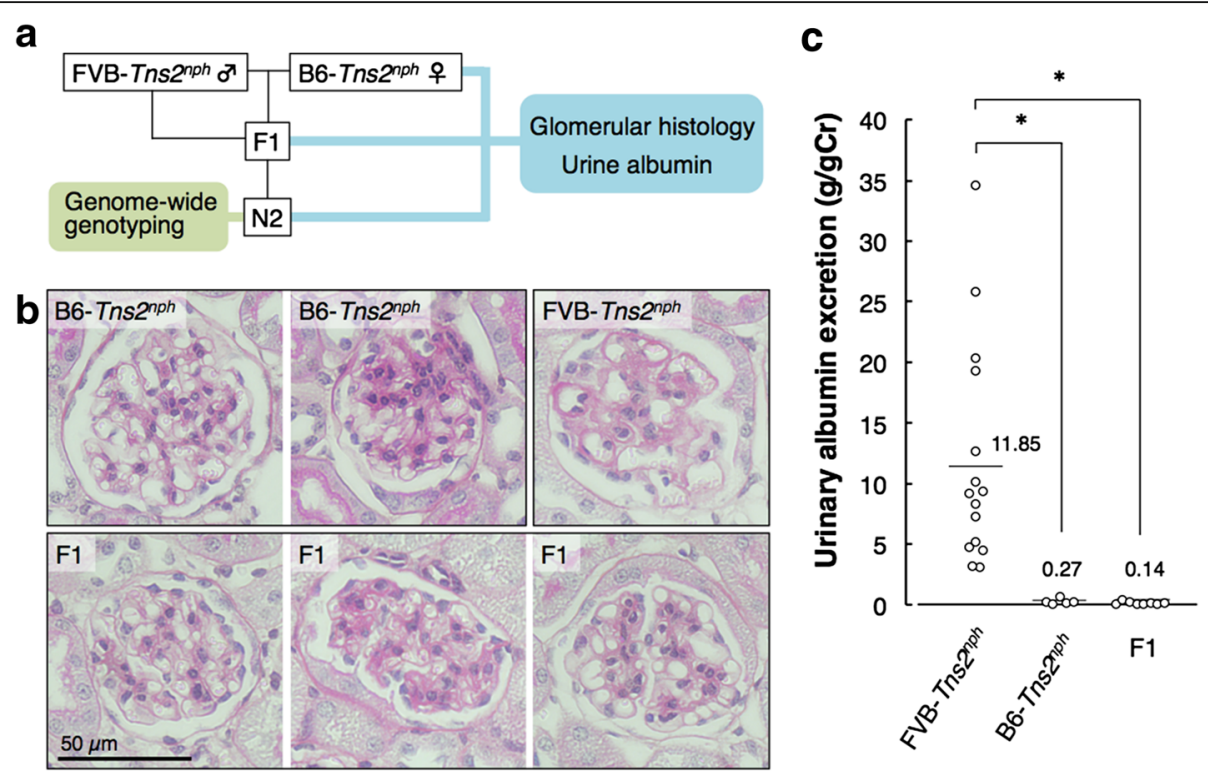

Fig. 1 Glomerular histology and urinary albumin excretion in Tns2-deficient B6, FVB and F1 mice. (a) Schema of the study. b Representative glomerular structures in PAS staining. In B6-Tns $2^{\text {nph }}$ mice, most glomeruli were normal (left panel), while some glomeruli showed a slight increase in mesangial matrix (right panel). In FVB-Tns $2^{\text {nph }}$ mice, many glomeruli showed marked expansion of the mesangial matrix, marked thickening of the GBM and abnormal dilation of the capillary lumen. In F1 mice, there were normal glomeruli (left panel), glomeruli with mesangial expansion (middle panel) and glomeruli with GBM thickening (right panel). c Urinary albumin excretion in FVB-Tns2 $2^{\text {nph }}(n=15)$, B6-Tns $^{\text {nph }}(n=5)$ and F1 $(n=8)$ mice. Values represent the mean. Asterisk, $P<0.01$ 


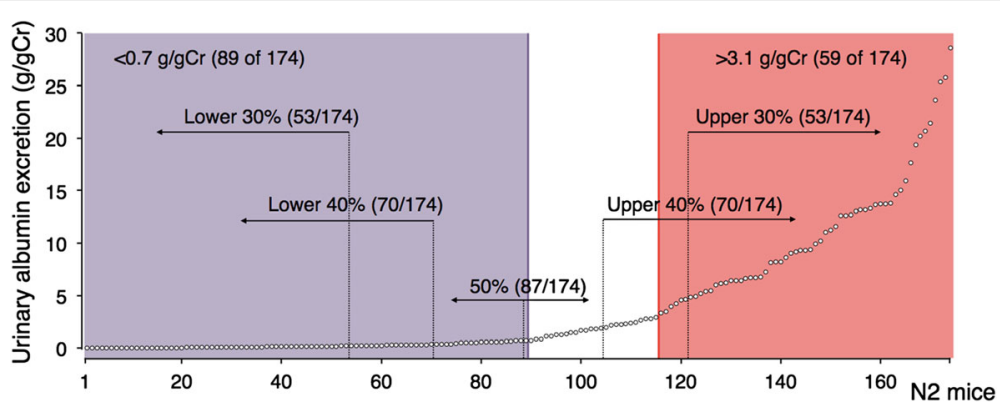

Fig. 2 Distribution of urinary albumin excretion in N2 mice. Vertical lines illustrated in the figure represent the borders of the phenotype definitions for marker-trait association test. $n=174$

room was air-conditioned at $22 \pm 4{ }^{\circ} \mathrm{C}$, maintained at $40-60 \%$ relative humidity, and mice were maintained under a $12 \mathrm{~h}$ light-dark cycle. A standard laboratory diet, CE-2 (CLEA Japan, Tokyo, Japan), and tap water were provided ad libitum.

\section{Genotyping}

Genome-wide genotyping of the Tns $2^{n p h}$ N2 mice was performed using 99 polymorphic markers (Additional file 1: Table S1). The Tyr locus was genotyped by coat color, and the other loci were genotyped using polymerase chain reaction (PCR)-based method. Genomic deoxyribonucleic acid was extracted from ear tissue obtained during ear punching for identification. PCR was performed with the following thermal cycling: initial denaturation at $95{ }^{\circ} \mathrm{C}$ for $3 \mathrm{~min}$; 10 cycles of $95{ }^{\circ} \mathrm{C}$ for $10 \mathrm{~s}, 65{ }^{\circ} \mathrm{C}$ for $30 \mathrm{~s}$ (reduced by $-1{ }^{\circ} \mathrm{C} /$ cycle), and $72{ }^{\circ} \mathrm{C}$ for $10 \mathrm{~s}$; and 30 cycles of $95{ }^{\circ} \mathrm{C}$ for $10 \mathrm{~s}, 55^{\circ} \mathrm{C}$ for $30 \mathrm{~s}$, $72{ }^{\circ} \mathrm{C}$ for $10 \mathrm{~s}$. Newly designed PCR primers are listed in the Additional file 1: Table S2.

\section{Measurement of urinary albumin excretion}

Urine samples were collected from 8-week-old mice by gentle manual compression of the abdomen. To measure albumin, urine samples were diluted with sample buffer containing $2 \%$ sodium dodecyl sulfate (SDS), $5 \%$ ß-mercaptoethanol, 10\% glycerol, $60 \mathrm{mM}$ Tris-HCL (pH 6.8) and bromophenol blue, and heated at $95{ }^{\circ} \mathrm{C}$ for $5 \mathrm{~min}$. Samples containing $1 \mu \mathrm{L}$ of urine were applied to $10 \%$ SDS-polyacrylamide gel electrophoresis. As a positive control, $5 \mu \mathrm{g}$ of bovine serum albumin was loaded simultaneously. The gel was fixed and stained with Coomassie brilliant blue (CBB; Nacalai Tesque, Kyoto, Japan) according to manufacturer's instructions, and scanned using a standard commercial scanner. CBB-stained urinary albumin was quantified by the ImageJ gel analysis program (http://imagej.net/). Urinary creatinine was measured using a creatinine colorimetric assay kit (Cayman chemical, Michigan, USA) according to manufacturer's instructions. The urinary albumin excretion was normalized against the urinary creatinine. Urine collection was

Table 1 Relationship between albuminuria and genotype for genetic markers on chromosomes 2 and 10

\begin{tabular}{|c|c|c|c|c|c|c|c|c|c|c|}
\hline \multirow[t]{2}{*}{ Chromosome } & \multirow[t]{2}{*}{ Marker } & \multirow{2}{*}{$\begin{array}{l}\text { Position } \\
\text { (cM) }\end{array}$} & \multicolumn{4}{|l|}{$X^{2}(P)$} & \multicolumn{4}{|c|}{ Student $-\mathrm{T}(\mathrm{P})^{\mathrm{c}}$} \\
\hline & & & $\overline{M i n} / \operatorname{Max}^{a}$ & $50 \%^{b}$ & $40 \%^{b}$ & $30 \%^{b}$ & Min/Max & $50 \%$ & $40 \%$ & $30 \%$ \\
\hline 2 & D2Mit398 & 61.76 & 0.19 & 0.062 & 0.052 & 0.20 & 0.48 & 0.47 & 0.40 & 0.31 \\
\hline 2 & rs214164633 & 65.66 & 0.16 & 0.080 & 0.075 & 0.20 & 0.26 & 0.25 & 0.24 & 0.18 \\
\hline 2 & rs234188972 & 70.98 & 0.18 & 0.067 & 0.062 & 0.24 & 0.14 & 0.16 & 0.14 & 0.10 \\
\hline 2 & rs247793542 & 75.41 & 0.64 & 0.36 & 0.30 & 0.84 & 0.25 & 0.23 & 0.26 & 0.24 \\
\hline 2 & rs 228447345 & 81.91 & 0.65 & 0.28 & 0.44 & 1 & 0.42 & 0.45 & 0.49 & 0.49 \\
\hline 10 & D10Mit298 & 2.78 & 0.58 & 0.42 & 0.60 & 0.48 & 0.43 & 0.47 & 0.36 & 0.29 \\
\hline 10 & D10Mit3 & 16.53 & 0.010 & 0.053 & 0.086 & 0.04 & 0.0099 & 0.0084 & 0.012 & 0.013 \\
\hline 10 & D10Mit197 & 35 & 0.049 & 0.059 & 0.059 & 0.10 & 0.0078 & 0.011 & 0.012 & 0.014 \\
\hline 10 & rs259766385 & 40.66 & 0.0022 & 0.0039 & 0.0068 & 0.0065 & 0.00038 & 0.00098 & 0.00074 & 0.00061 \\
\hline 10 & rs239646663 & 43.64 & 0.0043 & 0.0099 & 0.0040 & 0.019 & 0.00053 & 0.00076 & 0.0010 & 0.0015 \\
\hline 10 & D10Mit233 & 61.58 & 0.054 & 0.092 & 0.042 & 0.079 & 0.14 & 0.17 & 0.20 & 0.15 \\
\hline
\end{tabular}

${ }^{a}$ The N2 mice with urinary albumin excretion larger than $3.1 \mathrm{~g} / \mathrm{gCr}$, the minimum value in FVB-Tns2 ${ }^{\text {nph }}$ mice, were defined as the FVB type (affected) and the N2 mice with urinary albumin excretion less than $0.7 \mathrm{~g} / \mathrm{gCr}$, the maximum value in B6- Tns2 ${ }^{\text {nph }}$ mice, were defined as the B6 type (not affected)

${ }^{\mathrm{b}}$ The upper $\mathrm{X} \%$ and lower $\mathrm{X} \%$ of N2 mice in urinary albumin excretion were defined as the FVB type (affected) and the B6 type (not affected), respectively

'Urinary albumin excretion in N2 mice was compared between those with B6/FVB and FVB/FVB genotypes at the genetic markers 
Table 2 Relationship between albuminuria and combined genotypes of chromosomes 2 and 10 (rs259766385)

\begin{tabular}{|c|c|c|c|c|c|c|c|c|c|c|}
\hline \multirow[t]{2}{*}{ Chromosome } & \multirow[t]{2}{*}{ Marker } & \multirow{2}{*}{$\begin{array}{l}\text { Position } \\
\text { (cM) }\end{array}$} & \multicolumn{4}{|l|}{$X^{2}(P)$} & \multicolumn{4}{|c|}{ Student- $T(P)^{c}$} \\
\hline & & & $\overline{M i n} / M_{a x}^{a}$ & $50 \%{ }^{b}$ & $40 \%^{b}$ & $30 \%^{b}$ & Min/Max & $50 \%$ & $40 \%$ & $30 \%$ \\
\hline 2 & D2Mit398 & 61.76 & 0.003 & 0.0019 & 0.0049 & 0.014 & 0.055 & 0.078 & 0.075 & 0.027 \\
\hline 2 & rs214164633 & 65.66 & 0.0013 & 0.0012 & 0.0027 & 0.046 & 0.0016 & 0.0045 & 0.0028 & 0.0010 \\
\hline 2 & rs234188972 & 70.98 & 0.0013 & 0.00090 & 0.0032 & 0.0097 & 0.00021 & 0.0015 & 0.00083 & 0.00025 \\
\hline 2 & rs 247793542 & 75.41 & 0.0063 & 0.0054 & 0.012 & 0.024 & 0.0020 & 0.0070 & 0.0042 & 0.0013 \\
\hline 2 & $r s 228447345$ & 81.91 & 0.0039 & 0.0021 & 0.012 & 0.10 & 0.0089 & 0.028 & 0.025 & 0.015 \\
\hline
\end{tabular}

${ }^{a}$ The N2 mice with urinary albumin excretion larger than $3.1 \mathrm{~g} / \mathrm{gCr}$, the minimum value in FVB-Tns $2^{\text {nph }}$ mice, were defined as the FVB type (affected) and the N2 mice with urinary albumin excretion less than $0.7 \mathrm{~g} / \mathrm{gCr}$, the maximum value in B6- $\mathrm{Tns} 2^{\text {nph }}$ mice, were defined as the B6 type (not affected)

${ }^{\mathrm{b}}$ The upper X\% and lower X\% of N2 mice in urinary albumin excretion were defined as the FVB type (affected) and the B6 type (not affected), respectively

'Urinary albumin excretion in the homogeneic genotype, in which both markers were FVB/FVB genotype, were compared with those in the heterogeneic genotype, in which at least one of the markers was B6/FVB genotype

performed twice over a three-day period, and the measured urinary albumin excretion was averaged for each mouse.

\section{Histology}

Kidneys from 8 -week-old mice were fixed with $4 \%$ paraformaldehyde (PFA) at $4{ }^{\circ} \mathrm{C}$ overnight. The PFA-fixed paraffin sections ( $2 \mu \mathrm{m}$ thick) were subjected to normal histological processes and stained with periodic acidSchiff (PAS) solution.

\section{Marker-trait association test}

N2 mice were divided into two groups, affected and not affected, according to their urinary albumin excretion. With reference to the distribution of urinary albumin excretion observed in FVB-Tns $2^{n p h}$ and B6-Tns $2^{n p h}$ mice, we defined the $\mathrm{N} 2$ mice with urinary albumin excretion larger than the minimum value in FVB-Tns $2^{n p h}$ mice as the FVB type (affected with albuminuria) and the N2 mice with urinary albumin excretion less than the maximum value in B6-Tns $2^{n p h}$ mice as the B6 type (not affected with albuminuria). Another way of division was to define the upper $30 \%$ or $40 \%$ or $50 \%$ of N2 mice as the FVB type and the lower $30 \%$ or $40 \%$ or $50 \%$ of N2 mice as the B6 type. A chi-square test was applied to detect marker-trait associations.

\section{Statistics}

Data are expressed as means \pm standard deviation. Student's $t$-test and Dunnett's multiple comparison test were used for comparisons of two or more than two independent groups, respectively. A $p$ value $<0.05$ was considered statistically significant. Dunnett's multiple comparison test was carried out using GraphPad Prism 5 software (MDF, Tokyo, Japan).

\section{Results}

Phenotype of Tns2-deficient B6, FVB and (B6 $x$ FVB) $F_{1}$ mice Consistent with previous reports [12-15], FVB-Tns $2^{\text {nph }}$ mice showed albuminuria (urinary albumin excretion,
$11.8 \pm 9.2 \mathrm{~g} / \mathrm{gCr}$ ) and severe glomerular injuries, such as marked expansion of the mesangial matrix, marked thickening of the GBM and abnormal dilation of capillary lumen, whereas B6-Tns $2^{n p h}$ mice did not show either albuminuria (urinary albumin excretion, $0.26 \pm 0$. $25 \mathrm{~g} / \mathrm{gCr}$ ) or glomerular injury, apart from a slight increase in the mesangial matrix in some glomeruli (Fig. 1b, c). To assess the mode of inheritance of traits for resistance to Tns2 dysfunction, F1 mice were subjected to similar analyses at 8 weeks. The structures of some glomeruli in the F1 mice were nearly normal (Fig. 1b); however, unlike that in B6-Tns $2^{\text {nph }}$ mice, a focal thickening of the GBM was also observed in F1 mice (Fig. 1b), suggesting that F1 mice exhibited an intermediate phenotype for glomerular injury caused by Tns2 dysfunction. On the other hand, the urinary albumin excretion of F1 mice $(0.14 \pm 0.13 \mathrm{~g} / \mathrm{gCr})$ was approximately equal to that of B6-Tns $2^{\text {nph }}$ mice (Fig. 1c),

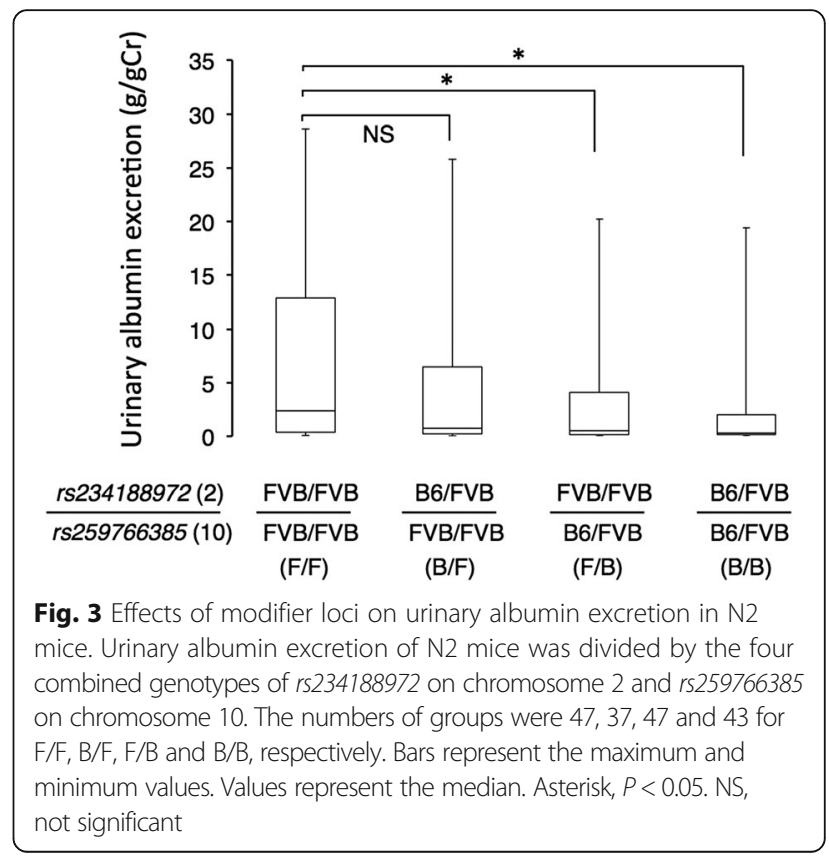




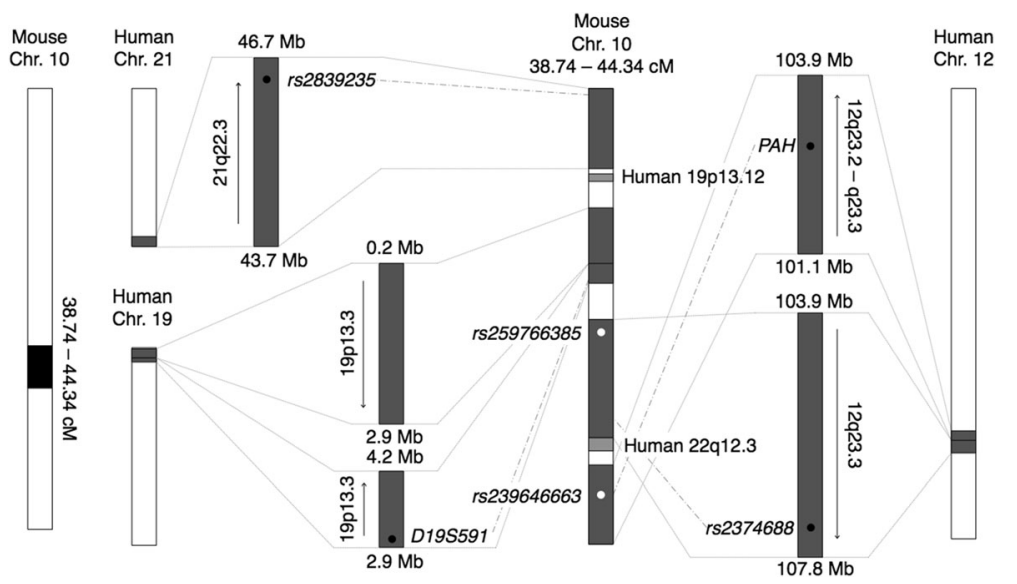

Fig. 4 Schematic representation of the genomic region of mouse chromosome 10 at 38.74-44.34 CM and the homologous regions in human. The genomic regions of mouse chromosome 10 at 38.74-44.34 cM are syntenic to human 12q23.2 - q23.3, 19p13.3, 19p13.12, 21q22.3 and 22q13.3. White dots represent mouse genetic markers used in this study: rs 259766385 and rs 239646663 are the peak markers of Tpir2. Black dots represent human genetic markers: rs 2374688 and rs 2839235 are the single nucleotide polymorphisms associated with urinary albumin excretion and glomerular filtration rate in human, respectively [21]; and PAH and D19S591 (also known as GATA44F10) are the genetic markers associated with urinary albumin excretion in patients with essential hypertension [22]. Chr., chromosome

suggesting that resistance to albuminuria caused by Tns 2 dysfunction is inherited as a dominant trait in F1 mice. Although the glomerular injuries may afford a sensitive reflection of the severity of renal disease caused by Tns2 dysfunction, we considered that urinary albumin excretion is a better indicator of resistance to Tns2 dysfunction than is glomerular injury, which is complicated and difficult to quantify.

\section{Genome-wide linkage analysis in N2 backcross mice}

As F1 mice as well as B6-Tns $2^{n p h}$ mice exhibited a suppressive phenotype, we backcrossed the F1 mouse onto the FVB-Tns $2^{n p h}$ mouse to identify the modifier loci related to resistance to Tns2 dysfunction. We then performed genome-wide genotyping of the resultant N2 mice ( $n=174$ ) and subsequent phenotyping for albuminuria. N2 mice showed a continuous spectrum of values for urinary albumin excretion and did not simply segregate into the B6- and FVB-Tns $2^{n p h}$ types according to whether or not they were affected with albuminuria (Fig. 2). Therefore, with reference to the distribution of urinary albumin excretion observed in FVB-Tns $2^{n p h}$ (3.12-34.6 g/ $\mathrm{gCr})$ and $\mathrm{B} 6-\mathrm{Tns} 2^{\text {nph }}$ mice $(0.052-0.69 \mathrm{~g} / \mathrm{gCr})$, we defined the N2 mice with urinary albumin excretion larger than 3. $1 \mathrm{~g} / \mathrm{gCr}$ (59 of 174) as the FVB type (affected) and the N2 mice with urinary albumin excretion less than $0.7 \mathrm{~g} / \mathrm{gCr}$ (89 of 174) as the B6 type (not affected). In the N2 mice as defined above, marker-trait associations were detected on chromosome 10. The genetic markers rs 259766385 and $r s 239646663$ on chromosome 10 showed a statistical significant relationship with albuminuria (Table 1). In addition, the analyses using different phenotype definitions (e.g., the upper $40 \%$ and lower $40 \%$ of $\mathrm{N} 2$ mice) found suggestive signals on chromosome 2 (Table 1). Furthermore, the combined rs259766385 genotypes and the genetic markers on chromosome 2 provided a more significant $p$ value in comparison with the chi-square test for each marker (Table 2). Table 2 shows a comparison between the two combined genotypes; one in which both the markers are homogeneic, the other in which at least one of the markers is heterogeneic. In Fig. 3, the variations in urinary albumin excretion were compared among the four combined genotypes for rs 234188972 on chromosome 2 and rs259766385 on chromosome 10. Urinary albumin excretion in both groups with allelic heterogeneity at $r s 259766385$ (F/B and B/B groups) was significantly lower than that in the group carrying $\mathrm{FVB} / \mathrm{FVB}$ genotypes at the two markers (F/F group) (Fig. 3). The median value for urinary albumin excretion in the group carrying B6/ FVB and FVB/FVB genotypes at rs234188972 and rs259766385, respectively (B/F group, $0.71 \mathrm{~g} / \mathrm{gCr}$ ), was approximately equal to those of the $\mathrm{F} / \mathrm{B}$ and $\mathrm{B} / \mathrm{B}$ groups ( 0.52 and $0.32 \mathrm{~g} / \mathrm{gCr}$, respectively) and appeared lower than that of the F/F group $(2.4 \mathrm{~g} / \mathrm{gCr})$, although no significant difference was detected between the $B / F$ and $F / F$ groups (Fig. 3).

\section{Discussion}

In this study, to identify the modifier loci in podocyte alterations caused by Tns2 dysfunction, we assessed the relationship between albuminuria phenotype and genotype in backcrosses between resistant B6-Tns $2^{\text {nph }}$ and susceptible FVB-Tns $2^{n p h}$ mice. Initially, we demonstrated that F1 mice exhibited an intermediate phenotype for glomerular injury between resistant B6 and susceptible FVB types, while little of no urinary 
albumin was detected in both the F1 mice and B6-Tns $2^{n p h}$ mice. The results indicated that heterozygous alleles at the modifier loci show an intermediate phenotype for podocyte injury, leading to an increase in albumin leakage through the glomerular filtration barrier. However, F1 mice showed a resistant B6-type phenotype for albuminuria, probably due to the proximal tubular reabsorption of albumin [20].

Subsequently, F1 mice were backcrossed to FVB-Tns $2^{n p h}$ mice. The N2 backcrosses showed a wide spectrum of urinary albumin excretion ranging from B6 type to FVB type, and including intermediate values. These values indicated that some N2 mice exhibited podocyte and glomerular injuries that were more severe than those of F1 mice but milder than those of FVBTns $2^{n p h}$ mice. These injuries are expected to lead to greater albumin leakage, which cannot be reabsorbed by the proximal tubules, resulting in a continuous spectrum of values for urinary albumin excretion in $\mathrm{N} 2$ mice. These results also indicate that there are multiple modifier loci for podocyte injury caused by Tns2 dysfunction. However, a QTL analysis of urinary albumin excretion failed to identify any modifier loci (data not shown). We assumed that this is because that the intended modifier effects are hindered due to a wide spectrum of values for urinary albumin excretion in affected mice themselves (see Fig. 1c). Therefore, we simply segregated N2 mice into two groups, affected and not affected, and examined the specific relationship between genotype and phenotype using chi-square test. Then, a marker-trait association test suggested the existence of two loci, a significant locus on chromosome 10 and a suggestive locus on chromosome 2, whose peak marker rs234188972 is included in Tpir (57.65-78. $72 \mathrm{cM})$. B6 homozygous alleles of Tpir exerted a protective effect against podocyte alterations and reduced urinary albumin excretion in affected mice to approximately $50 \%$ [18]. In this study, B6 heterozygous alleles of the suggestive locus on chromosome 2 were observed to partially alleviate podocyte injury, based on the results shown in Fig. 3. We, therefore, speculated that a causative gene at the suggestive locus is identical to Tpir, and we designated this suggestive locus as Tpir1.

As the protective effect of the B6 allele of Tpir was insufficient in comparison with that of the $\mathrm{B} 6$ genetic background itself, our previous study predicted the existence of an as yet unidentified major modifier locus [18], which actually corresponds to the significant locus identified on chromosome 10. B6 heterozygous alleles of the significant locus markedly reduced urinary albumin excretion in affected mice. Furthermore, B6 heterozygous alleles of both Tpir1 and the significant locus exerted a protective effect on albuminuria at levels close to that of the $\mathrm{B} 6$ genetic background. We, therefore, designated the significant locus as Tpir2. No genes located around Tpir1 and Tpir2 are known to be involved in nephropathy.

The genomic region of chromosome 10 at 38.74-44. $34 \mathrm{cM}$ including rs259766385, the peak marker of Tpir2, consists of multiple syntenic regions on human chromosomes 12, 19, 21 and 22. It is notable that the syntenic regions on human 12q23.2 - q23.3 and 19p13.3, and 21q22.3 include the genetic markers associated with urinary albumin excretion (PAH, rs2374688, D19S591) and glomerular filtration rate (rs2839235) (Fig. 4) [21, 22]. It is interesting that the multiple genetic markers associated with urinary albumin excretion or glomerular filtration rate on the different human chromosomes are located on a single syntenic 5.6-cM interval (38.74-44.34 cM) of mouse chromosome 10, albeit including a gene-rich region (39.72cM) that encompasses 233 genes. This suggests that there might be multiple modifier genes on Tpir2. The Tpir2 genes located on syntenic human 12q23.2 - q23.3, 19p13.3 and $21 \mathrm{q} 22.3$ are shown in the Additional file 1: Table S3.

\section{Conclusions}

Our present analysis identified two genetic regions associated with podocyte injury induced by Tns 2 dysfunction, Tpir1 and Tpir2, which are on chromosomes 2 and 10, respectively. Tpir1, which is considered to be identical to Tpir, the genetic region identified by our previous study, is insufficient to explain the resistance of the B6 genetic background to Tns2 dysfunction, while Tpir2 is a major modifier locus for this resistance. The combined protective effect of the B6 alleles of Tpir1 and Tpir2 was comparable to that of the $\mathrm{B} 6$ genetic background itself. The identification of these modifier genes is expected to help elucidate the mechanism underlying podocyte injury.

\section{Additional file}

Additional file 1: Table S1. Genomic positions of polymorphic genetic markers between B6 and FVB strains. Table S2. Sequences of the PCR primers used to identify polymorphic indel mutations between B6 and FVB strains. Table S3. Genes located on syntenic human 12q23.2 - q23.3, 19p13.3 and 21q22.3. (XLSX $28 \mathrm{~kb})$

\section{Abbreviations}

CBB: Coomassie brilliant blue; CKD: Chronic kidney disease; CM: Centimorgan; GBM: Glomerular basement membrane; gCr: Gram creatinine; PAS: Periodic acid-Schiff; PCR: Polymerase chain reaction; PFA: Paraformaldehyde; PTB: Phosphotyrosine binding; PTP: Protein tyrosine phosphatase;

QTL: Quantitative trait loci; Rho-GAP: Rho GTPase-activating protein;

SDS: Sodium dodecyl sulfate; SH2: Src homology 2

\section{Acknowledgements}

We wish to thank our lab members for their helpful discussions.

\section{Funding}

This work was supported by the Ministry of Education, Culture, Sports, Science and Technology (MEXT), Glant-in-Aid for Scientific Research, KAKENHI (16K16606) to HS and KAKENHI (20302614) to NS. The funders had no role in the design of the study and collection, analysis, decision to publish, interpretation of data or preparation of the manuscript. 


\section{Availability of data and materials}

All the data supporting the conclusions of the study are included in the manuscript and the additional file.

\section{Author's contributions}

HS and NS conceived and designed the work. YT and SO performed the experiments and analyzed the data. HS and YT drafted the manuscript. NS revised the manuscript. All authors reviewed the manuscript. All authors have read and approved the final version of the manuscript.

\section{Ethics approval and consent to participate}

All research was conducted according to the Regulations for the Care and Use of Laboratory Animals of Kitasato University. The animal experimentation protocol was approved by the President of Kitasato University based on the judgment made by the Institutional Animal Care and Use Committee of Kitasato University (Approval ID: No. 15-167).

\section{Competing interests}

The authors declare no competing interests.

\section{Publisher's Note}

Springer Nature remains neutral with regard to jurisdictional claims in published maps and institutional affiliations.

Received: 2 November 2017 Accepted: 3 April 2018

Published online: 10 April 2018

\section{References}

1. Haynie DT. Molecular physiology of the tensin brotherhood of integrin adaptor proteins. Proteins. 2014;82(7):1113-27.

2. Chen H, Duncan IC, Bozorgchami H, Lo SH. Tensin1 and a previously undocumented family member, tensin2, positively regulate cell migration. Proc Natl Acad Sci U S A. 2002;99(2):733-8.

3. Hafizi $S$, Alindri F, Karlsson R, Dahlbäk B. Interaction of Axl receptor tyrosine kinase with C1-TEN, a novel C1 domain-containing protein with homology to tensin. Biochem Biophys Res Commun. 2002;299(5):793-800.

4. Cho AR, Uchio-Yamada K, Torigai T, Miyamoto T, Miyoshi I, Matsuda J, Kurosawa T, Kon Y, Asano A, Sasaki N, et al. Deficiency of the tensin2 gene in the ICGN mouse: an animal model for congenital nephrotic syndrome. Mamm Genome. 2006;17(5):407-16.

5. Nishino T, Sasaki N, Chihara M, Nagasaki K, Torigoe D, Kon Y, Agui T. Distinct distribution of the tensin family in the mouse kidney and small intestine. Exp Anim. 2012:61(5):525-32.

6. Hong SY, Shih YP, Sun P, Hsieh WJ, Lin WC, Lo SH. Down-regulation of tensin2 enhances tumorigenicity and is associated with a variety of cancers. Oncotarget. 2016;7(25):38143-53.

7. Hafizi S, Ibraimi F, Dahlbäk B. C1-TEN is a negative regulator of the Akt/PKB signal transduction pathway and inhibits cell survival, proliferation, and migration. FASEB J. 2005;19(8):971-3.

8. Marusugi K, Nakano K, Sasaki H, Kimura J, Yanobu-Takanashi R, Okamura T, Sasaki N. Functional validation of tensin2 SH2-PTB domain by CRISPR/Cas9mediated genome editing. J Vet Med Sci. 2016;78(9):1413-20.

9. Ogura A, Fujimura H, Asano T, Koura M, Naito I, Kobayashi Y. Early ultrastructural glomerular alterations in neonatal nephrotic mice (ICGN strain). Vet Pathol. 1995;32(3):321-3.

10. Uchio K, Manabe N, Tamura K, Miyamoto M, Yamaguchi M, Ogura A Yamamoto $Y$, Miyamoto $\mathrm{H}$. Decreased matrix metalloproteinase activity in the kidneys of hereditary nephrotic mice (ICGN strain). Nephron. 2000;86(2):145-51.

11. Mizuno S, Kurosawa T, Matsumoto K, Mizuno-Horikawa Y, Okamoto M, Nakamura T. Hepatocyte growth factor prevents renal fibrosis and dysfunction in a mouse model of chronic renal disease. J Clin Invest. 1998;101(9):1827-34.

12. Sasaki H, Marusugi K, Kimura J, Kitamura H, Nagasaki K, Torigoe D, Agui T, Sasaki N. Genetic background-dependent diversity in renal failure caused by the tensin2 gene deficiency in the mouse. Biomed Res. 2015;36(5):323-30.

13. Uchio-Yamada K, Sawada K, Tamura K, Katayama S, Monobe Y, Yamamoto Y, Ogura A, Manabe N. Tenc1-deficient mice develop glomerular disease in a strain-specific manner. Nephron Exp Nephrol. 2013;123(3-4):22-33.

14. Uchio-Yamada K, Monobe Y, Akagi K, Yamamoto Y, Ogura A, Manabe N. Tensin2-deficient mice on FVB/N background develop severe glomerular disease. J Vet Med Sci. 2016;78(5):811-8.
15. Nishino T, Sasaki N, Nagasaki K, Ahmad Z, Agui T. Genetic background strongly influences the severity of glomerulosclerosis in mice. J Vet Med Sci. 2010;72(10):1313-8.

16. Nishino T, Sasaki N, Nagasaki K, Ichii O, Kon Y, Agui T. The 129 genetic background affects susceptibility to glomerulosclerosis in tensin2-deficient mice. Biomed Res. 2012;33(1):53-6.

17. Sasaki H, Sasaki N, Nishino T, Nagasaki K, Kitamura H, Torigoe D, Agui T. Quantitative trait loci for resistance to the congenital nephropathy in tensin 2-deficient mice. PLoS One. 2014;9(6):e99602.

18. Sasaki H, Kimura J, Nagasaki K, Marusugi K, Agui T, Sasaki N. Mouse chromosome 2 harbors genetic determinants of resistance to podocyte injury and renal tubulointerstitial fibrosis. BMC Genet. 2016;17(1):69.

19. Mason RM, Wahab NA. Extracellular matrix metabolism in diabetic nephropathy. J Am Soc Nephrol. 2003;14(5):1358-73.

20. Birn H, Christensen El. Renal albumin absorption in physiology and pathology. Kidney Int. 2006;69(3):440-9.

21. Hwang SJ, Yang Q, Meigs JB, Pearce EN, Fox CS. A genome-wide association for kidney function and endocrine-related traits in the NHLBI's Framingham heart study. BMC Med Genet. 2007;8(Suppl 1):S10.

22. Freedman BI, Beck SR, Rich SS, Heiss G, Lewis CE, Turner S, Province MA Schwander KL, Arnett DK, Mellen BG, et al. A genome-wide scan for urinary albumin excretion in hypertensive families. Hypertension. 2003:42(3):291-6.

\section{Ready to submit your research? Choose BMC and benefit from:}

- fast, convenient online submission

- thorough peer review by experienced researchers in your field

- rapid publication on acceptance

- support for research data, including large and complex data types

- gold Open Access which fosters wider collaboration and increased citations

- maximum visibility for your research: over $100 \mathrm{M}$ website views per year

At BMC, research is always in progress.

Learn more biomedcentral.com/submissions 